\title{
Current usage of stereotactic body radiotherapy for oligometastatic prostate cancer in Korea: patterns of care survey (KROG 19-08)
}

\author{
Sun Hyun Bae', Won Il Jang', Hyun-Cheol Kang ${ }^{3}$, Young Il Kim ${ }^{4}$, Yong Ho Kim ${ }^{5}$, Woo Chul Kim ${ }^{6}$, \\ Hee Kwan $\mathrm{Lee}^{7}$, Jin Ho Kim ${ }^{3}$
}

${ }^{1}$ Department of Radiation Oncology, Soonchunhyang University College of Medicine, Bucheon, Republic of Korea; ${ }^{2}$ Korea Institute of Radiological and Medical Sciences, Seoul, Republic of Korea; ${ }^{3}$ Seoul National University College of Medicine, Seoul, Republic of Korea; ${ }^{4}$ Chungnam National University School of Medicine, Daejeon, Republic of Korea; ${ }^{5}$ International St. Mary's Hospital, Catholic Kwandong University College of Medicine,

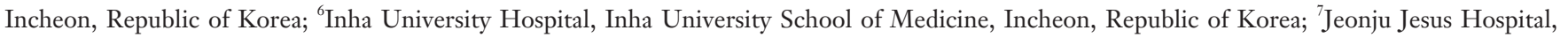
Jeollabuk-do, Republic of Korea

Contributions: (I) Conception and design: SH Bae, JH Kim; (II) Administrative support: SH Bae; (III) Provision of study materials or patients: SH Bae, WI Jang; (IV) Collection and assembly of data: SH Bae, HC Kang, YI Kim; (V) Data analysis and interpretation: SH Bae, YH Kim, WC Kim, HK Lee, JH Kim; (VI) Manuscript writing: All authors; (VII) Final approval of manuscripts: All authors.

Correspondence to: Jin Ho Kim, MD, PhD. Department of Radiation Oncology, Seoul National University College of Medicine, 101, Daehak-ro, Jongno-gu, Seoul 03080, Republic of Korea. Email: jinho.kim.md@gmail.com.

Background: Growing evidence suggests that metastasis-directed therapy and/or prostate-directed therapy may benefit patients with oligometastatic prostate cancer (OMPC). Stereotactic body radiotherapy (SBRT) is increasingly used to treat oligometastases in various cancers. The purpose of this study was to investigate the current patterns of curative-intent SBRT for OMPC in Korea.

Methods: A 20-item questionnaire was sent to 326 radiation oncologists in 93 institutions in Korea. Only 1 physician per institution was required to complete the survey. Subsequently, the second survey consisting of 3 clinical scenarios was sent to 64 physicians with clinical experience in SBRT: case 1, cT4N0M1 (direct invasion to two pelvic bones); case 2, cT2N0M1 (three bone metastases); and case 3, solitary spine metastasis after radical prostatectomy.

Results: Seventy-six physicians from 93 institutions (82\%) answered the first survey. The multidisciplinary team approach was practiced in 16 institutions (21\%). Most physicians (75\%) agreed on the definition of oligometastases as limited lesions and/or organs $\leq 5: 25 \%$ agreed with low-volume disease according to CHAARTED trial. During the last year, 49 physicians (64\%) treated OMPC patients with curative intent. Sixty four physicians (84\%) had a clinical experience with SBRT: 48 (75\%) stated that both dose and fraction number should be considered when defining SBRT, whereas others (25\%) stated that only fraction size should be considered. Fifty-five faculties (86\%) answered the second survey. Physicians agreed with oligometastases in $89 \%$ for case 1 , in $80 \%$ for case 2 , and in $100 \%$ for case 3 . The rate of SBRT application was the highest in case 3 (70\%).

Conclusions: There was diversity in the patterns of SBRT for OMPC in Korea. Additional prospective studies are necessary to strengthen evidence regarding role of SBRT in OMPC.

Keywords: Korea; oligometastases; prostate cancer (PC); stereotactic body radiotherapy (SBRT); survey

Submitted Mar 08, 2021. Accepted for publication Jul 07, 2021.

doi: $10.21037 /$ atm-21-1116

View this article at: https://dx.doi.org/10.21037/atm-21-1116 


\section{Introduction}

Cancer is one of the major leading causes of death worldwide, and prostate cancer (PC) is the second most common malignancies among men worldwide. In Korea, PC is the fourth common cancer, and its incidence has been continuously increasing since 1999: approximately $10 \%$ of patients are diagnosed at distant metastatic stage (1). In patients with metastatic hormone sensitive PC, the standard treatment is androgen deprivation therapy (ADT) alone or in combination with apalutamide, abiraterone, or docetaxel with palliative intent $(2,3)$. Unfortunately, most patients develop castration-resistant PC (CRPC) within 5 years of diagnosis, and CRPC is considered a lethal disease due to the lack of optimal treatment, although several new drugs for CRPC have shown survival benefits (4). Against this background, recent some prospective and retrospective studies reported that local treatments such as metastasis-directed therapy (MDT) and/or prostate-directed therapy (PDT) improved the survival in oligometastatic prostate cancer (OMPC), suggesting a shift in the management of metastatic PC (5-7).

The concept of oligometastases was first proposed by Hellman and Weichselbaum in 1995. For certain tumors, the anatomy and physiology may limit or concentrate theses metastases to a single or a limited number of organs, and local modalities such as radiotherapy (RT) can improve the patients' survival and have a curative potential (8). However, the definition of oligometastases remains ambiguous. Therefore, different study groups set their own arbitrary criteria, based on the total number of metastatic lesions and/ or organs. Although a new technology, stereotactic body radiotherapy (SBRT), allows the delivery of high radiation doses, no consensus has been reached on the universally sufficient radiation doses to ablate oligometastases (9). The emerging interest in OMPC led to the increasing application of SBRT with a potentially curative intent; its patterns of practice vary widely in the absence of high-level evidence.

Therefore, the Korean Stereotactic Radiosurgery Group of the Korean Society for Radiation Oncology (KOSRO) conducted a national patterns-of-care survey to better understand the patterns of curative-intent SBRT practice for OMPC in Korea. We present the following article in accordance with the SURGE reporting checklist (available at https://dx.doi.org/10.21037/atm-21-1116).

\section{Methods}

A 20-item questionnaire was sent by an e-mail to 326 radiation oncologists, who are full members of KOSRO, at
93 institutions in Korea in October 2019. The questionnaire was based on their clinical experience with OMPC and SBRT. Only 1 physician per institution was required to complete the survey sent by e-mail within 1 month. We selected one survey by order of arrival when we received multiple replies from 1 institution at the same time. Subsequently, the second survey was sent to 64 radiation oncologists in 64 institutions who had clinical experience in SBRT. The second survey consisted of questions regarding three OMPC cases. The complete survey was returned by e-mail within 1 month. The full contents of the two surveys are available in Appendix 1 and 2. In the event of nonresponse, the respondents were contacted by telephone and sent e-mails in order to achieve a response rate of more than $80 \%$. The study was conducted in accordance with the Declaration of Helsinki (as revised in 2013). This study was approved by the institutional review board of Soonchunhyang University College of Medicine, Bucheon (IRB No. 2019-08-023-001). The need for written informed consent from the participants was waived because this study was a survey that used retrospective data of patients who were treated and we anonymized and de-identified all records and information prior to analysis so as not to infringe any patients' rights. This study was also conducted under the authorization and cooperation of the Korea Radiation Oncology Group (KROG 19-08).

\section{Results}

\section{Clinical experience (number of respondents $=76$ )}

Seventy-six physicians (82\%) from 93 institutions responded to the first survey. Sixteen physicians have been working as radiation oncologists for $<5$ years after completing residency, 19 for 5-9 years, 18 for $10-19$ years, and 23 for $\geq 20$ years. Approximately $51 \%$ of these physicians are working in tertiary referral hospitals, and $46 \%$ are working in secondary care hospitals. The multidisciplinary team approach for PC patients has been adopted in 16 institutions (21\%): regularly in 7 institutions and irregularly in 9 institutions. There is a radiation oncologist as a specialist for urology in $75 \%$ of respondents. The remaining institutions have either only one radiation oncologist $(13 \%)$ or a non-urologic cancer specialist (12\%). The annual per-physicians cases of radical RT for PC were as follows: $\leq 5$ cases in 4 physicians (5\%), $6-10$ in $6(8 \%)$, $11-30$ in 27 (35\%), 31-50 in 22 (29\%), 51-100 in $11(15 \%)$, and 101-300 in $6(8 \%)$. We presented the physicians' working period and the annual per-physicians cases at Figure 1. The association between the physicians' working period and 


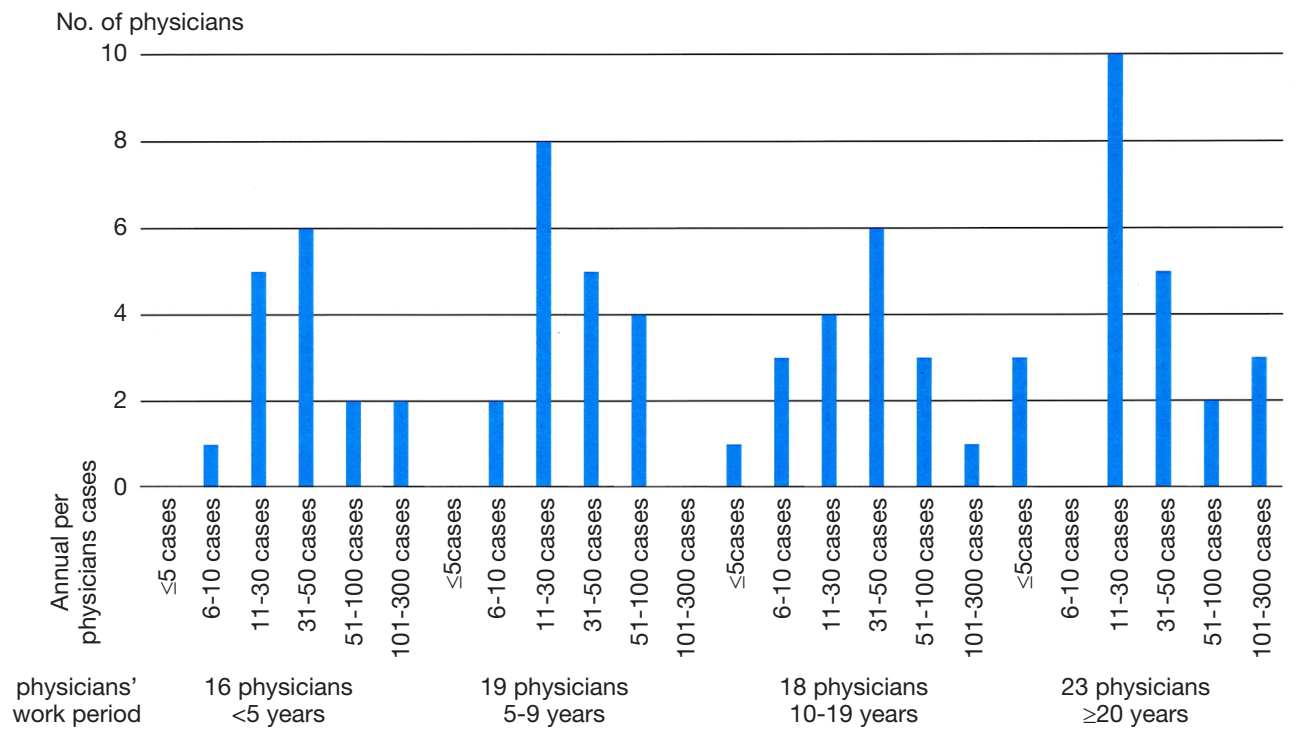

Figure 1 The annual per-physicians cases of radical radiotherapy for prostate cancer according to physicians' work period.

Table 1 The definition of oligometastatic prostate cancer $(n=76)$

\begin{tabular}{lll}
\hline Definition & N & $\%$ \\
\hline 1. Low-volume according to CHAARTED trial ${ }^{a}$ & 19 & 25 \\
2. Limited lesions and/or organs & 57 & 75 \\
Number of lesions & & \\
1 & 1 & \\
$2-3$ & 29 \\
$4-5$ & 27 \\
Number of organs & \\
1 & 17 \\
$2-3$ & 32 \\
5 & 8 \\
\hline
\end{tabular}

a, means all other patients except for patients with a high volume of metastases, which was defined by the presence of visceral metastases or four or more bone lesions with at least one beyond the vertebral bodies and pelvis. CHAARED, Chemohormonal Therapy Versus Androgen Ablation Randomized Trial for Extensive Disease in Prostate Cancer.

SBRT experience is shown in Figure S1.

\section{View on oligometastases (number of respondents $=76$ )}

Fifty-seven respondents (75\%) agreed with the definition of OMPC as 5 or less metastatic lesions and/or organs $\leq 5$,
Table 2 Required imaging studies to establish oligometastatic prostate cancer $(\mathrm{n}=76)$

\begin{tabular}{|c|c|c|}
\hline Image work up ${ }^{a}$ & $\mathrm{~N}$ & $\%$ \\
\hline 1. Prostate MRI & 76 & 100 \\
\hline 2. Whole body bone scan & 69 & 91 \\
\hline 3. AP CT/chest CT & 49 & 65 \\
\hline 4. Spine MRI & 30 & 40 \\
\hline 5. FDG PET-CT & 28 & 37 \\
\hline 6. Choline or PSMA PET-CT & 5 & 7 \\
\hline 7. Bone SPECT & 1 & 1 \\
\hline \multicolumn{3}{|c|}{$\begin{array}{l}\text { a, the respondents selected multiple answer. MRI, magnetic } \\
\text { resonance imaging; CT, computed tomography; FDG, } \\
{ }^{18} \text { F-fluorodeoxyglucose; PET, positron emission tomography; } \\
\text { PSMA, prostate-specific membrane antigen, SPECT, single- } \\
\text { photon emission computed tomography. }\end{array}$} \\
\hline
\end{tabular}

whereas only 19 (25\%) responded that oligometastases constituted a low metastatic burden based on the CHAARTED trial criteria (Table 1) (10). Physicians rely on diverse imaging studies to establish a diagnosis of OMPC (Table 2). Twenty-seven radiation oncologists (35\%) had no experience treating OMPC patients with a curative intent. For the remaining 49 physicians, the annual number of OMPC patients referred for RT with curative intent are $\leq 5$ in $49 \%$ (37 physicians), $6-10$ in 11\% (8), 11-20 in 4\% (3), and $\geq 21$ in $1 \%$ (1). All 49 physicians agreed on the use of 
Table 3 Target volumes and timing of radiotherapy (RT) for initially diagnosed oligometastatic prostate cancer $(n=76)$

\begin{tabular}{lcc}
\hline & N & $\%$ \\
\hline Target volume & 27 & 36 \\
1. No case & 0 & 0 \\
2. No RT & 4 & 5 \\
3. Prostate only & 5 & 7 \\
4. Up to 1-2 metastases only & 3 & 4 \\
5. Up to 3 metastases only & 0 & 0 \\
6. Up to 4-5 metastases only & 14 & 18 \\
7. Prostate and up to 1-2 metastases & 16 & 21 \\
8. Prostate and up to 3 metastases & 6 & 8 \\
9. Prostate and up to 4-5 metastases & 1 & 1 \\
10. Case by case & & \\
RT timing & & \\
1. No case & 27 & 36 \\
2. Concurrent with ADT $\leq 1$ month & 10 & 13 \\
3. Delay after neoadjuvant ADT & 23 & 30 \\
2-8 months & & \\
4. As soon as possible because \\
patients are consulted for RT after
\end{tabular}

ADT, androgen deprivation therapy.
RT for OMPC. The target volumes and timing of RT for patients who were initially diagnosed with OMPC varied (Table 3).

\section{SBRT experience (number of respondents $=64$ )}

Among the 76 respondents, 12 (16\%) without SBRT experience were excluded from further survey. The remaining 64 radiation oncologists with SBRT experience continued the survey. Forty-eight physicians (75\%) stated that both dose and fraction number should be considered when defining SBRT, whereas 16 (25\%) stated that the only fraction size should be considered. The detailed numbers are presented in Figure 2. At present, the National Health Insurance Service in Korea provides reimbursements for the cost of SBRT of up to 4 fractions only, regardless of the actually delivered fractions. Six radiation oncologists (9\%) are contented with the current reimbursement schemes, whereas 58 physicians (91\%) opined that the insurance should cover more than 4 fractions.

\section{SBRT for OMPC (number of respondents $=64$ )}

In the past year, the most common fractionation schemes of PDT for OMPC were as follows: hypofractionated RT in $27(42 \%)$, SBRT in $6(9 \%)$, conventional fractionated RT in $5(8 \%)$, and case-by-case basis in $5(8 \%)$ [21 physicians (33\%) had no case]. The most common fractionation

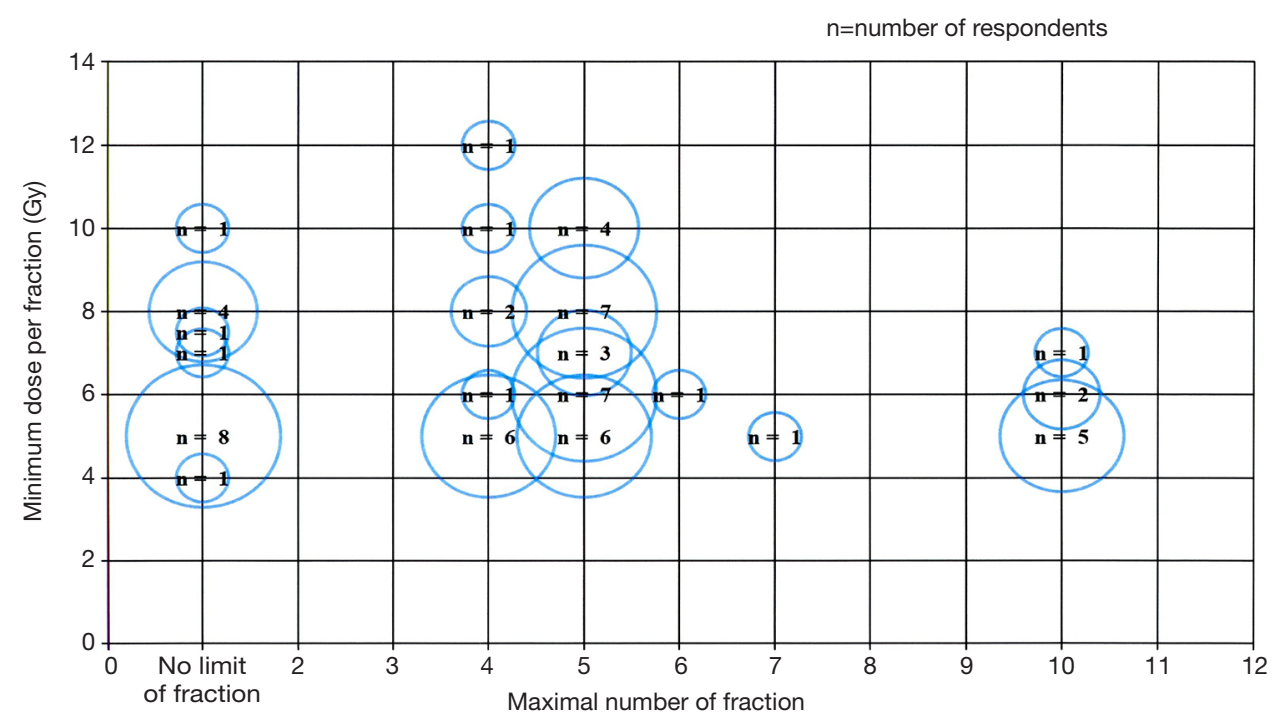

Figure 2 Definition of stereotactic body radiotherapy according to fraction and dose. 
Table 4 Prostate immobilization tool, target localization, and image guided radiotherapy (IGRT) workflow during stereotactic body radiotherapy (SBRT) $(\mathrm{n}=64)$

\begin{tabular}{|c|c|c|}
\hline & $\mathrm{N}$ & $\%$ \\
\hline \multicolumn{3}{|l|}{ Immobilization tool for prostate SBRT } \\
\hline 1. No application of SBRT for prostate & 41 & 64 \\
\hline 2. No use & 15 & 23 \\
\hline 3. Endorectal balloon & 7 & 11 \\
\hline \multicolumn{3}{|l|}{ Target localization during SBRT ${ }^{\mathrm{a}}$} \\
\hline 1. Orthogonal KV radiographs & 9 & 14 \\
\hline 2. Orthogonal MV radiographs & 3 & 5 \\
\hline 3. Fluoroscopy & 0 & 0 \\
\hline \multicolumn{3}{|l|}{ IGRT workflow during SBRT } \\
\hline 1. Image $\rightarrow$ Correction $\rightarrow$ Treatment $(T x)$ & 28 & 44 \\
\hline 2. Image $\rightarrow$ Correction $\rightarrow \mathrm{Tx} \rightarrow$ Image after Tx & 2 & 3 \\
\hline 3. Image $\rightarrow$ Correction $\rightarrow \mathrm{Tx} \rightarrow$ Image during $\mathrm{Tx} \rightarrow \mathrm{Tx}$ & 4 & 6 \\
\hline 4. Image $\rightarrow$ Correction $\rightarrow \mathrm{Tx} \rightarrow$ Image during $\mathrm{Tx} \rightarrow \mathrm{Tx} \rightarrow$ Image after Tx & 0 & 0 \\
\hline 5. Image $\rightarrow$ Correction $\rightarrow$ Image $\rightarrow$ Tx & 18 & 28 \\
\hline 6. Image $\rightarrow$ Correction $\rightarrow$ Image $\rightarrow \mathrm{Tx} \rightarrow$ Image after Tx & 4 & 6 \\
\hline 7. Image $\rightarrow$ Correction $\rightarrow$ Image $\rightarrow \mathrm{Tx} \rightarrow$ Image during $\mathrm{Tx} \rightarrow \mathrm{Tx}$ & 5 & 8 \\
\hline
\end{tabular}

${ }^{a}$, the respondents selected multiple answer. KV, kilovoltage; MV, megavoltage; CBCT, cone beam computed tomography; MRI, magnetic resonance imaging.

schemes for MDT were as follows: SBRT in 26 (40\%), hypofractionated RT in 11 (17\%), case-by-case basis in $4(6 \%)$, conventional fractionated RT in $1(2 \%)$, and no MDT for OMPC in $1(2 \%)$. The respondent stated that the use of SBRT for OMPC was hampered by the lack of suitable patients for SBRT ( $\mathrm{n}=36,56 \%)$, preference for other fractionation $(\mathrm{n}=21,33 \%)$, and reimbursement issues $(n=9,14 \%)$, when allowed to select multiple answers. The pattern of PDT using SBRT is presented in Table 4. Most radiation oncologists did not use any immobilization device for prostate SBRT, and the preferred method for target localization was kilovoltage or megavoltage cone beam computed tomography. All physicians obtained the images before every treatment, and the image registration workflow during and after treatment varied (Table 4).

\section{Clinical cases (number of respondents $=55$ )}

Of 64 physicians with SBRT experience, 55 (86\%) responded to the second survey. Details of the three clinical cases are shown in Figure 3. They generally agreed that the three cases were categorized as OMPC to varying degrees: 49 respondents for case 1, 44 for case 2, and 55 for case 3 . For case 1, 25 respondents selected to treat the whole pelvis, including regional lymph node (LN) chains and pelvic bone metastases, and 20 treated both the prostate and metastatic lesions. For case 2, 24 the respondents selected to treat the prostate and metastatic lesions, while 16 treated the whole pelvis. For case 3, all physicians treated only the metastatic lesion, but the target volume varied, as shown in Figure 4. The most preferred fractionation scheme for PDT was 

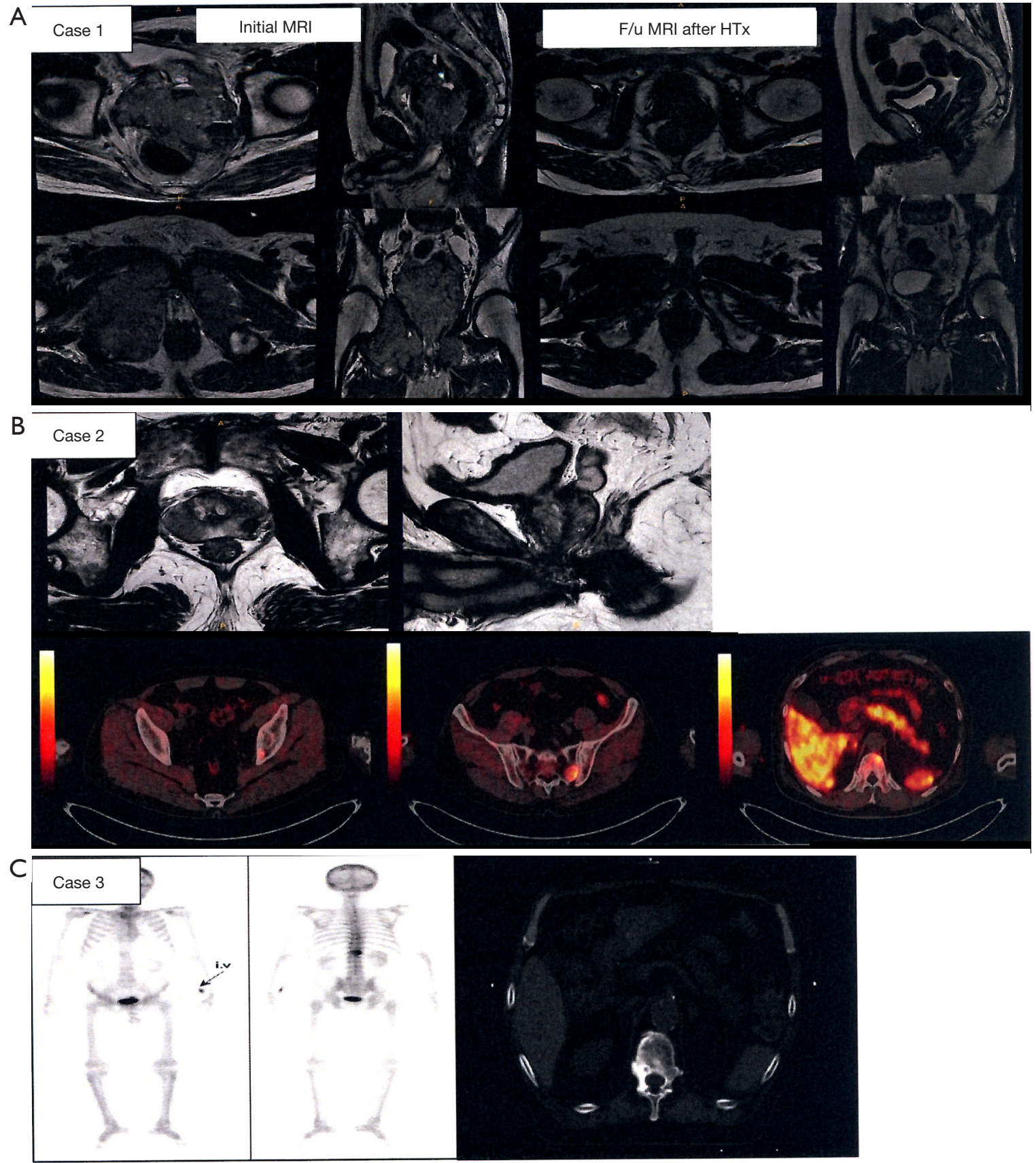

Figure 3 Three clinical cases which was presented in the second questionnaire survey (A) Case 1: a 69-year-old patient with prostate cancer with direct invasion to two pelvic bones at the right acetabulum and pubic bone [Gleason score $(\mathrm{G} / \mathrm{S})=4+4$, cT4N0M1, initial prostate-specific antigen (PSA) $>1,000 \mathrm{ng} / \mathrm{mL}$. He was referred for radiotherapy (RT) when the level of PSA decreased to $<0.03 \mathrm{ng} / \mathrm{mL}$ after undergoing androgen deprivation therapy (ADT) for 1 year. (B) Case 2: a 64-year-old patient with prostate cancer with three bone metastases at the left acetabulum, left sacral alar, and $11^{\text {th }}$ thoracic (T11) spine (ECOG 0, G/S = 4+4, cT2N0M1, initial PSA 162.88 ng/mL). He was referred for RT when the level of PSA decreased to $41.40 \mathrm{ng} / \mathrm{mL}$ after receiving 1 cycle of ADT. (C) Case 3: a 65-year-old patient with prostate cancer with solitary metastases in the T12 spine after undergoing ADT, radical prostatectomy, salvage RT to prostate bed, and cytotoxic chemotherapy (ECOG 1, G/S = 5+4, PSA $7.89 \mathrm{ng} / \mathrm{mL}$ ). 

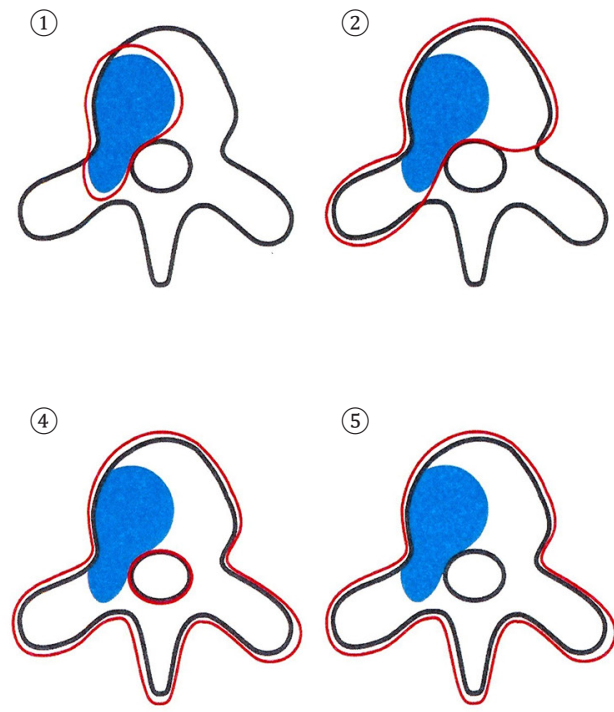

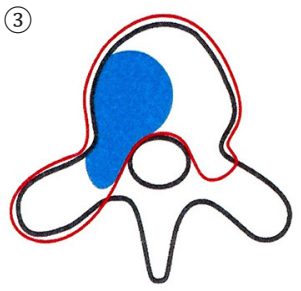

\begin{tabular}{ccc}
\hline Target volume & $\mathrm{N}$ & $\%$ \\
\hline (1) & 1 & 2 \\
$(2)$ & 18 & 34 \\
(3) & 21 & 40 \\
(4) & 4 & 7 \\
(5) & 9 & 17 \\
(6) & 0 & 0 \\
\hline
\end{tabular}

(6)

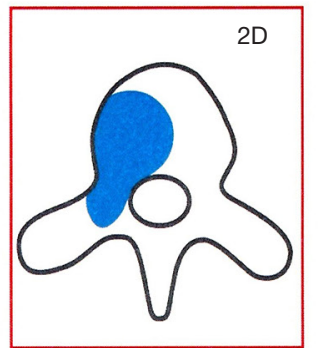

Figure 4 Target volume for spinal metastases for case 3: A total of 53 physicians selected the target volume, except for two who did not apply radiotherapy in this case.

hypofractionated RT for PTT, whereas that for MDT varied according to the site of metastases. Hypofractionated RT is preferred for pelvic bone metastases, while SBRT is preferred for spinal metastases. Other details are summarized in Table 5 .

\section{Discussion}

Hellman and Weichselbaum first proposed oligometastases as an intermediate state between a localized disease and a systemically metastatic disease (8). This includes comprehensively synchronous or metachronous metastases, and controlled or uncontrolled primary tumors, regardless of the number of lesions, if all viable tumors can be treated with local modalities. Afterwards Niibe et al. (11) proposed a new definition of oligorecurrence: one to several distant metastases/recurrences (usually one) in one to several organs (usually one) with controlled primary tumor. Recently, the EORTC and ESTRO group subclassified oligometastases into synchronous oligometastases, metachronous oligorecurrence, and metachronous oligoprogression (12). Although these subclassifications would provide a clear system and reflect the fundamental biology, the exact definition of limited metastases has not yet been determined. The first phase I and randomized phase II studies, which applied SBRT as MDT for OMPC, included patients with $1-3$ metastatic lesions $(13,14)$. On the other hand, a phase III study reported that SBRT as a
PDT for synchronous metastatic PC improved the overall survival of patients with a low metastatic burden according to the CHAARTED trial (15). Recent ESTRO-ASTRO consensus recommends that oligometastases can be defined as presence of 1-5 metastatic lesions (16). Meanwhile, ongoing prospective studies on OMPC can allow 5-10 metastatic lesions or unlimited metastases (17). Our survey reported that most physicians agreed that oligometastases involved a limited number of metastases, but the allowed number varied. There is a need to reach a consensus for the standardized and harmonized practice for OMPC among radiation oncologists in Korea.

A higher RT dose improves disease control in patients with localized PC, and at least 75.6 Gy conventionally fractionated RT has been established as the modern standard treatment (18). Based on the radiobiologic sensitivity to hypofractionation of PC, patient convenience, and health care costs, non-inferiority phase 3 randomized trials have confirmed the safety and efficacy of hypofractionation compared with conventional fractionation (19). Therefore, hypofractionated RT is recommended as a standard of care across all risk groups (20). SBRT is an extreme form of hypofractionation, which utilizes either a single dose or a small number of fractions; many prospective studies reported that SBRT showed similar toxicity and noninferior disease control compared with conventional or hypofractionated RT (21-23). Nonetheless, treatment 
Table 5 Details for each case $(n=55)$

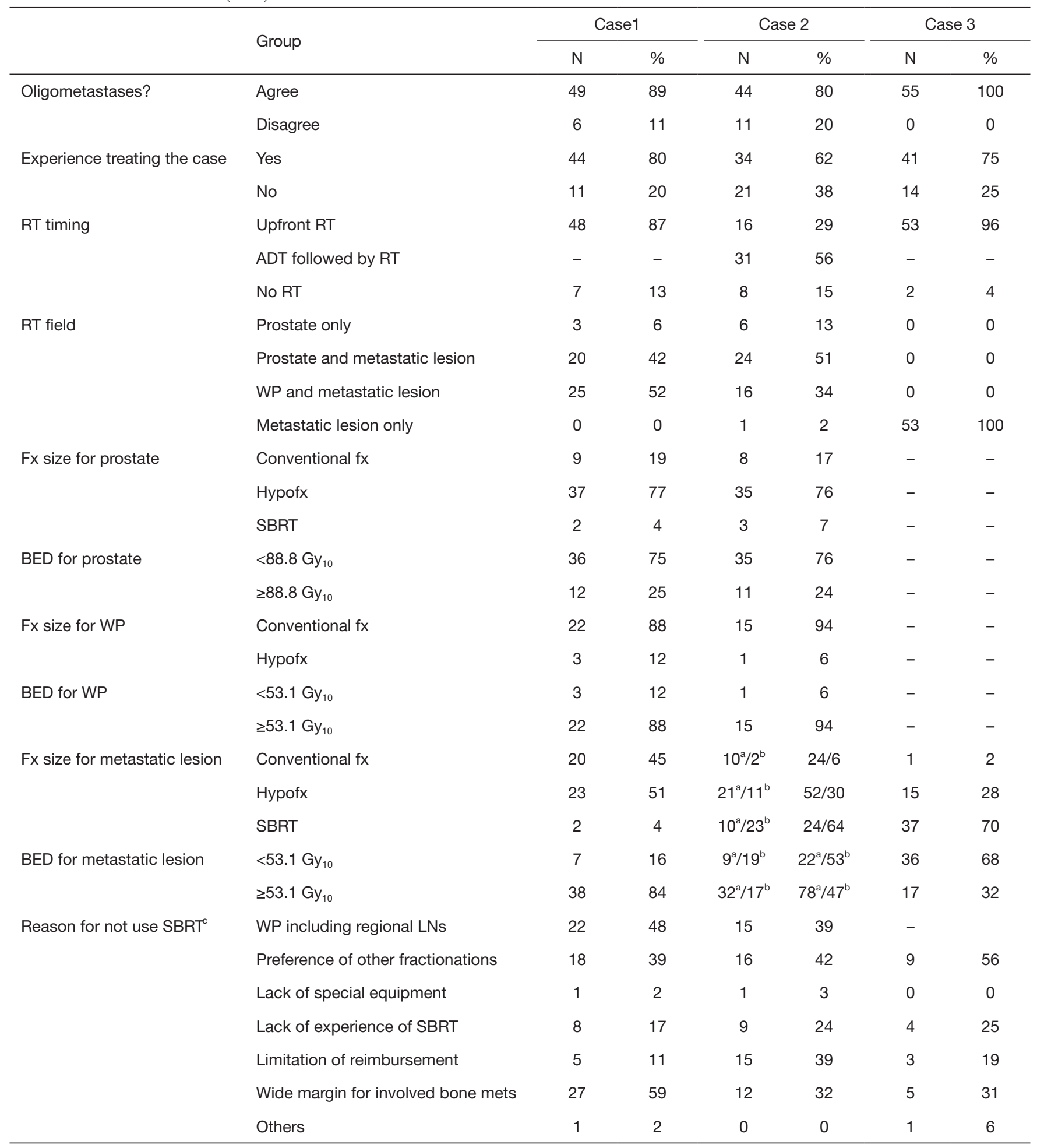

a, means pelvic bone metastases; ${ }^{b}$, means T11 spine metastases; ${ }^{\text {, }}$, the respondents selected multiple answer. RT, radiotherapy; ADT, androgen deprivation therapy; WP, whole pelvis including regional lymph nodes (LNs); Fx, fraction; Hypofx, hypofractionation; SBRT, stereotactic body radiotherapy; BED, biologically effective dose when $\alpha / \beta$ was assumed to be 10 Gy. 
guidelines recommend SBRT for patients with low-risk $\mathrm{PC}$ and is considered as an alternative treatment option for those with intermediate-risk and high-risk PC (20). $\mathrm{RT}$ as a PDT for OMPC was assessed in two prospective studies $(15,24)$. The HORRAD trial initiated study with 70 Gy in 35 fractions and additionally permitted 55.76 Gy in 19 fractions. The authors pointed out that the total dose was lower than the currently applied for PC, and it was considered as a limitation of the study. The STAMPEDE trial used 36 Gy in 6 fractions or 55 Gy in 20 fractions because the standard regimen of 74 Gy in 37 fractions would be too burdensome for patients with metastatic PC. In the current survey, most physicians (42\%) selected hypofractionated RT as PDT for OMPC, and only $9 \%$ used SBRT. This partially affected by the insurance coverage. Since the inclusion of intensity-modulated RT (IMRT) in the health insurance system in 2015, IMRT use increased dramatically in Korea, and PC is the third most common cancer treated with IMRT $(25,26)$. Whereas, the national insurance policy for SBRT remained unchanged and only covers at total $\leq 4$ fractions, thus making it difficult to apply the standard number of SBRT fractions (5-6 fractions) in patients with PC. Although SBRT is an attractive modality for delivering higher radiation doses and reducing the overall treatment time, SBRT as a PDT for OMPC should be carefully performed in clinical trials due to the low quality of evidence and limited insurance resource in Korea.

SBRT as an MDT for oligometastases has been evaluated in various organ sites from different types of primary cancers. A recent multi-institutional randomized phase II study of 1-5 oligometastases from any type of primary cancer (OMPC, 16\%) compared the standard therapy with SBRT and that without SBRT as MDT (27). SBRT is associated with a 13 -months increase in the overall survival and the doubling of progression-free survival, but the risk of toxicity increased, including a 5\% risk of grade 5 toxicity. Phase I and II studies on SBRT as MDT for OMPC showed that ADT-free survival was longer with MDT and the quality of life was maintained after SBRT $(13,14)$. Other prospective and retrospective studies using SBRT as MDT for OMPC reported a promising local control rate of $80-100 \%$ at 2 years (6). Although SBRT as an MDT for OMPC is effective, there is no consensus on the target volume and RT dose for metastatic lesions. Prostate cancer mainly metastasizes to the bone and LNs: bone metastases divide into spine and nonspine bone metastases. The RTOG 0631 phase II/III study was the first study to specifically describe the target volume for spinal metastases according to the extent of tumor (28). International Spine Radiosurgery Consortium Consensus published contouring guidelines for SBRT for spine metastases in more detail (29). Although there are detailed recommendations from expert consensus exist, contouring must be completed on a case-by-case basis with each case tailored to the patient's individual clinical situation and institutional infrastructure: Figure 4. reflects this potential variation in the clinical setting. For nonspine bone metastases, significant heterogeneity for contouring exists worldwide due to the absence of guidelines (30). For LN metastases, the optimal target volume between regional LN chains or affected LN only is unclear. One study reporting that two out of three OMPC patients treated with SBRT for pelvic $\mathrm{LN}$ relapsed in the nodes again might support the inclusion of all regional LN chains (31). Our study showed that physicians selected the regional LN chains on caseby-case basis. Ongoing multicenter, randomized, phase 2 PEACE V-STORM trial might yield clues to the potential benefit about elective nodal approach with whole pelvis RT as an alternative to focal SBRT in OMPC (32). In addition, several studies on MDT for OMPC with newer imaging modalities will help in the selection of optimal patients for SBRT (17). In terms of RT dose, most studies used 16-20 Gy in 1 fraction or 27 Gy in 3 fractions for bone metastases and lower doses for LN metastases $(6,9)$. The universally ablative dose should be clinically validated based on patients' outcomes by conducting further studies.

The current study has some limitations. First, respondents recollected SBRT experiences for OMPC from the past and recall bias may have occurred. Second, we used closedended questions and conducted descriptive analysis to get definite and vast information because little is unknown about practical patterns for SBRT for OMPC in Korea before this study. Further surveys composed of open-ended questions should be needed to reflect the accurate clinical practice. And last, the practical patterns of the respondents for OMPC may be different from those with no response. However, this survey may have representativeness because we have achieved response rate $>80 \%$ from all radiation oncologists in Korea ( $82 \%$ in the first survey and $86 \%$ in the second survey).

In conclusion, this is the first survey to present the practical patterns of SBRT for OMPC in Korea. Most physicians agreed on the definition of OMPC as limited lesions and/or organs, but different institutions provided their own arbitrary cutoffs. The definition of the fractionation scheme of SBRT differed among institutions. Although the target volume were various among physicians, SBRT was 
commonly used for spinal metastases. On the other hand, physicians preferred hypofractionated RT for the treatment of the prostate and nonspine bone metastases. Based on the findings of this survey, we should conduct additional prospective studies to standardize the practice and strengthen the evidence on the role of SBRT in OMPC.

\section{Acknowledgments}

Funding: This work was supported by the Soonchunhyang University Research Fund. No funding bodies had any role in study design, data collection and analysis, decision to publish, or preparation of the manuscript.

\section{Footnote}

Reporting Checklist: The authors have completed the SURGE reporting checklist. Available at https://dx.doi. org/10.21037/atm-21-1116

Data Sharing Statement: Available at https://dx.doi. org/10.21037/atm-21-1116

Conflicts of Interest: All authors have completed the ICMJE uniform disclosure form (available at https://dx.doi. org/10.21037/atm-21-1116). The authors have no conflicts of interest to declare.

Ethical Statement: The authors are accountable for all aspects of the work in ensuring that questions related to the accuracy or integrity of any part of the work are appropriately investigated and resolved. The study was conducted in accordance with the Declaration of Helsinki (as revised in 2013). This retrospective study was approved by the institutional review board of Soonchunhyang University College of Medicine, Bucheon (IRB No. 2019-08-023-001). Because of the retrospective nature, the requirement of written informed consent was waived.

Open Access Statement: This is an Open Access article distributed in accordance with the Creative Commons Attribution-NonCommercial-NoDerivs 4.0 International License (CC BY-NC-ND 4.0), which permits the noncommercial replication and distribution of the article with the strict proviso that no changes or edits are made and the original work is properly cited (including links to both the formal publication through the relevant DOI and the license). See: https://creativecommons.org/ licenses/by-nc-nd/4.0/.

\section{References}

1. Hong S, Won YJ, Park YR, et al. Cancer Statistics in Korea: Incidence, Mortality, Survival, and Prevalence in 2017. Cancer Res Treat 2020;52:335-50.

2. Chi KN, Agarwal N, Bjartell A, et al. Apalutamide for Metastatic, Castration-Sensitive Prostate Cancer. N Engl J Med 2019;381:13-24.

3. Mottet N, van den Bergh RCN, Briers E, et al. EAUESTRO-ESUR-SIOG Guidelines on Prostate Cancer. [Internet]. European Association of Urology.; Available online: https://uroweb.org/wp-content/uploads/EAUESUR-ESTRO-SIOG-Guidelines-on-Prostate-Cancerlarge-text-V2.pdf

4. Cornford P, Bellmunt J, Bolla M, et al. EAU-ESTROSIOG Guidelines on Prostate Cancer. Part II: Treatment of Relapsing, Metastatic, and Castration-Resistant Prostate Cancer. Eur Urol 2017;71:630-42.

5. Battaglia A, De Meerleer G, Tosco L, et al. Novel Insights into the Management of Oligometastatic Prostate Cancer: A Comprehensive Review. Eur Urol Oncol 2019;2:174-88.

6. Palacios-Eito A, Béjar-Luque A, Rodríguez-Liñán $M$, et al. Oligometastases in prostate cancer: Ablative treatment. World J Clin Oncol 2019;10:38-51.

7. Miura N, Pradere B, Mori K, et al. Metastasis-directed therapy and prostate-targeted therapy in oligometastatic prostate cancer: a systematic review. Minerva Urol Nefrol 2020;72:531-42.

8. Hellman S, Weichselbaum RR. Oligometastases. J Clin Oncol 1995;13:8-10.

9. Tree AC, Khoo VS, Eeles RA, et al. Stereotactic body radiotherapy for oligometastases. Lancet Oncol 2013;14:e28-37.

10. Sweeney CJ, Chen YH, Carducci M, et al. Chemohormonal Therapy in Metastatic Hormone-Sensitive Prostate Cancer. N Engl J Med 2015;373:737-46.

11. Niibe Y, Hayakawa K. Oligometastases and oligorecurrence: the new era of cancer therapy. Jpn J Clin Oncol 2010;40:107-11.

12. Guckenberger $M$, Lievens $Y$, Bouma $A B$, et al. Characterisation and classification of oligometastatic disease: a European Society for Radiotherapy and Oncology and European Organisation for Research and Treatment of Cancer consensus recommendation. Lancet Oncol 2020;21:e18-28.

13. Siva S, Bressel M, Murphy DG, et al. Stereotactic 
Abative Body Radiotherapy (SABR) for Oligometastatic Prostate Cancer: A Prospective Clinical Trial. Eur Urol 2018;74:455-62.

14. Ost P, Reynders D, Decaestecker K, et al. Surveillance or Metastasis-Directed Therapy for Oligometastatic Prostate Cancer Recurrence: A Prospective, Randomized, Multicenter Phase II Trial. J Clin Oncol 2018;36:446-53.

15. Parker CC, James ND, Brawley CD, et al. Radiotherapy to the primary tumour for newly diagnosed, metastatic prostate cancer (STAMPEDE): a randomised controlled phase 3 trial. Lancet 2018;392:2353-66.

16. Lievens Y, Guckenberger M, Gomez D, et al. Defining oligometastatic disease from a radiation oncology perspective: An ESTRO-ASTRO consensus document. Radiother Oncol 2020;148:157-66.

17. De Bruycker A, Tran PT, Achtman AH, et al. Clinical perspectives from ongoing trials in oligometastatic or oligorecurrent prostate cancer: an analysis of clinical trials registries. World J Urol 2021;39:317-26.

18. Royce TJ, Mavroidis P, Wang K, et al. Tumor Control Probability Modeling and Systematic Review of the Literature of Stereotactic Body Radiation Therapy for Prostate Cancer. Int J Radiat Oncol Biol Phys 2021;110:227-36.

19. Catton CN, Lukka H, Gu CS, et al. Randomized Trial of a Hypofractionated Radiation Regimen for the Treatment of Localized Prostate Cancer. J Clin Oncol 2017;35:1884-90.

20. NCCN. Prostate cancer (NCC Nguidelines version 2.2020). Available online: https://www.nccn.org/ professionals/physician_gls/pdf/prostate.pdf. 2020

21. Brand DH, Tree AC, Ostler P, et al. Intensity-modulated fractionated radiotherapy versus stereotactic body radiotherapy for prostate cancer (PACE-B): acute toxicity findings from an international, randomised, open-label, phase 3, non-inferiority trial. Lancet Oncol 2019;20:1531-43.

22. Widmark A, Gunnlaugsson A, Beckman L, et al. Ultrahypofractionated versus conventionally fractionated radiotherapy for prostate cancer: 5-year outcomes of the HYPO-RT-PC randomised, non-inferiority, phase 3 trial. Lancet 2019;394:385-95.

23. Jackson WC, Silva J, Hartman HE, et al. Stereotactic Body Radiation Therapy for Localized Prostate Cancer: A Systematic Review and Meta-Analysis of Over 6,000 Patients Treated On Prospective Studies. Int J Radiat Oncol Biol Phys 2019;104:778-89.
24. Boevé LMS, Hulshof MCCM, Vis AN, et al. Effect on Survival of Androgen Deprivation Therapy Alone Compared to Androgen Deprivation Therapy Combined with Concurrent Radiation Therapy to the Prostate in Patients with Primary Bone Metastatic Prostate Cancer in a Prospective Randomised Clinical Trial: Data from the HORRAD Trial. Eur Urol 2019;75:410-8.

25. Huh SJ, Park W, Choi DH. Recent trends in intensitymodulated radiation therapy use in Korea. Radiat Oncol J 2019;37:249-53.

26. Kim E, Jang WI, Kim MS, et al. Clinical utilization of radiation therapy in Korea, 2016. J Radiat Res 2020;61:249-56.

27. Palma DA, Olson R, Harrow S, et al. Stereotactic ablative radiotherapy versus standard of care palliative treatment in patients with oligometastatic cancers (SABRCOMET): a randomised, phase 2, open-label trial. Lancet 2019;393:2051-8.

28. Ryu S, Yoon H, Stessin A, et al. Contemporary treatment with radiosurgery for spine metastasis and spinal cord compression in 2015. Radiat Oncol J 2015;33:1-11.

29. Cox BW, Spratt DE, Lovelock M, et al. International Spine Radiosurgery Consortium consensus guidelines for target volume definition in spinal stereotactic radiosurgery. Int J Radiat Oncol Biol Phys 2012;83:e597-605.

30. Nguyen TK, Sahgal A, Dagan R, et al. Stereotactic Body Radiation Therapy for Nonspine Bone Metastases: International Practice Patterns to Guide Treatment Planning. Pract Radiat Oncol 2020;10:e452-60.

31. Decaestecker K, De Meerleer G, Lambert B, et al. Repeated stereotactic body radiotherapy for oligometastatic prostate cancer recurrence. Radiat Oncol 2014;9:135.

32. De Bruycker A, Spiessens A, Dirix P, et al. PEACE V - Salvage Treatment of OligoRecurrent nodal prostate cancer Metastases (STORM): a study protocol for a randomized controlled phase II trial. BMC Cancer 2020;20:406.

Cite this article as: Bae SH, Jang WI, Kang HC, Kim YI, Kim YH, Kim WC, Lee HK, Kim JH. Current usage of stereotactic body radiotherapy for oligometastatic prostate cancer in Korea: patterns of care survey (KROG 19-08). Ann Transl Med 2021;9(16):1291. doi: 10.21037/atm-21-1116 


\section{Appendix 1}

Stereotactic body radiotherapy (SBRT) for oligometastatic prostate cancer (OMPC)

Hospital: $\quad$ Name:

1. In what kind of institution are you currently working? ( )
(1) Primary- (2) Secondary-
(3) Tertiary health care hospital

2. How long have you been working as a radiation oncologist after residency? ( )
(1) $<5$ years
(2) 5-9 years
(3) $10-19$ years
(4) $\geq 20$ years

3. Is there a multidisciplinary team (MDT) approach for patients with prostate cancer in your institution? ( )

(1) Yes: Move to question 3-1. (2) No: Move to question 4.

3-1) Is the MDT meeting regularly performed? ( )

(1) Yes (2) No

3-2) How often do you have the MDT meetings? ( )
(1) Every week
(2) Every month
(3) About 2-3 times a month
(7) Others (Please specify)
(5) Every 3 months
(6) Every 4 months
(4) Every other month

4. Is there a radiation oncologist as a specialist for urology in your institution? ( )
(1) Yes
(2) No.
(3) No. I work alone.

5. How many prostate cancer patients do you treat with RT for a cure in the past year? ( )
(1) $\leq 5$ cases
(2) 6-10 cases
(3) $11-30$ cases
(4) 31-50 cases
(5) 51-100 cases
(6) 101-300 cases

6. What is your definition of oligometastases? ( )

(1) Number of lesions/number of organs (Please specify)

(2) Low-volume of metastatic disease according to the CHAARTED trial

7. Which imaging study do you consider to evaluate disease extent and metastases? ( ) (Please select all that apply)
(1) Prostate MRI
(2) Bone scan
(3) CT scan of abdomen and/or thorax
(4) Spine MRI
(5) FDG PET-CT
(6) Choline- or PSMA PET-CT

(7) Others (Please specify)

8. How many OMPC patients were referred for radical-intent RT in the past year? ( )
(1) No case
(2) $\leq 5$ cases
(3) 6-10 cases
(4) $11-20$ cases
(5) $\geq 21$ cases

9. What target volume do you treat for oligometastatic prostate cancer? ( )
(1) No case
(2) No RT
(3) Prostate only
(5) Up to 3 metastatic lesions only
(7) Prostate and up to 1-2 metastatic lesions
(9) Prostate and up to 4-5 metastatic lesions
(4) Up to 1-2 metastatic lesions only
(6) Up to 4-5 metastatic lesions only
(8) Prostate and up to 3 metastatic lesions
(10) Others (Please specify)

10. When do you apply RT for initially diagnosed OMPC patients? ( )
(1) No case
(2) Concurrent with androgen deprivation therapy (ADT): Start RT within 1 month after ADT.
(3) Neoadjuvant ADT 2-8 months: at__ months
(4) As soon as possible, because patients are consulted for RT $\geq 6$ months after ADT
(5) Others (Please specify)

11. Do you have an experience with SBRT to treat cancer patients? ( )

(1) Yes: Move to question 12.

(2) No: End the survey and thank you for your time.

12. What is your definition of SBRT? ( )

(1) ( ) Gy/fx, regardless of the number of fractions

(2) Above ( ) Gy and below ( ) fractions

13. Approximately, what percentage of OMPC patients, who were referred for RT, is treated with SBRT in the past year? 13-1-1) What is the application rate of SBRT for the primary lesion (prostate)? ( ) 
(1) No case No use of SBRT: application of conventional fraction (1.8-2 Gy/fx)

(3) No use of SBRT: application of hypofractionation (>2 Gy/fx)

(4) No use of SBRT: application of various fractionation scheme on case-by-case
(5) $1 \%-9 \%$
(6) $10 \%-40 \%$
(7) $41 \%-60 \%$
(8) $61 \%-99 \%$
(9) $100 \%$

13-1-2) What fractionation scheme do you most commonly utilize? ( Gy/ fx's)

13-2-1) What is the application rate of SBRT for oligometastatic lesions? ( )

(1) No case (2) No use of SBRT: application of conventional fraction (1.8-2 Gy/fx)

(3) No use of SBRT: application of hypofractionation $(>2 \mathrm{~Gy} / \mathrm{fx}$ )

(4) No use of SBRT: application of various fractionation scheme case by case
(5) $1 \%-9 \%$
(6) $10 \%-40 \%$
(7) $41 \%-60 \%$
(8) $61 \%-99 \%$
(9) $100 \%$

13-2-2) What fractionation scheme do you most commonly utilize?

( Gy/ fx's) to spine metastases

( Gy/ fx's) to other bone metastases

( Gy/ fx's) to lymph node metastases

( Gy/ fx's) to other metastases (Please specify the site)

14. What are reasons why it is difficult to use SBRT for OMPC patients? ( ) Please select all that apply.
(1) N/A: Always use SBRT.
(2) The lack of special equipment

(3) The lack of experience with using SBRT (4) The lack of
(5) Transfer of appropriate patients for SBRT to a larger hospital

(8) Others (Please specify)

(6) Preference for other fractionation scheme

(7) Insurance problems

15. The National Health Insurance Service in Korea has approved SBRT in cases of RT regimens using $\leq 4$ fractions applied to lesions within the body. Is it appropriate to limit the number of fractions? ( )
(1) Yes
(2) No: It is necessary to increase the number of fractions to

16. What treatment machine do you use for SBRT to a primary lesion (prostate)? ( ) Please select all that apply.
(1) No RT
(2) CyberKnife
(6) TrueBeam (Varian)
(3) RapidArc (Varian)
(7) Novalis (Varian)
(4) TomoTherapy
(5) Clinac iX (Varian)
(9) ViewRay ${ }^{\mathrm{TM}}$
(10) Proton
(11) Others (Please specify)
(8) VMAT (Elekta)

17. Do you use an immobilization tool for SBRT to the primary lesion (prostate)? ( )
(1) No use
(2) Yes: Use an endorectal balloon.
(3) Yes: Use rectal spacer.
(4) Others (Please specify)

18. What treatment machine do you use for SBRT to the oligometastatic lesions? ( ) Please select all that apply.
(1) No RT
(2) CyberKnife
(6) TrueBeam (Varian)
(10) Proton
(3) RapidArc (Varian)
(7) Novalis (Varian)
(4) TomoTherapy
(5) Clinac iX (Varian)
(9) ViewRay ${ }^{\mathrm{TM}}$
(11) Others (Please specify)
(8) VMAT (Elekta)

19. What target localization methods do you prefer for SBRT? ( )
(1) Orthogonal MV localization images
(4) $\mathrm{KV}$ or MV cone beam CT
(2) Orthogonal KV radiographs
(5) MRI
(3) Fluoroscopy
(6) Others (Please specify) _

20. In what order do you apply the image registration during SBRT? ( )
(1) Image $\rightarrow$ Correction $\rightarrow$ Treat
(2) Image $\rightarrow$ Correction $\rightarrow$ Treat $\rightarrow$ Image after $T x$
(3) Image $\rightarrow$ Correction $\rightarrow$ Treat $\rightarrow$ Image during $T_{x} \rightarrow$ Treat
(4) Image $\rightarrow$ Correction $\rightarrow$ Treat $\rightarrow$ Image during $\mathrm{Tx}_{\mathrm{x}} \rightarrow$ Treat $\rightarrow$ Image after $\mathrm{Tx}$
(5) Image $\rightarrow$ Correction $\rightarrow$ Image $\rightarrow$ Treat
(6) Image $\rightarrow$ Correction $\rightarrow$ Image $\rightarrow$ Treat $\rightarrow$ Image after Tx
(7) Image $\rightarrow$ Correction $\rightarrow$ Image $\rightarrow$ Treat $\rightarrow$ Image during $\mathrm{Tx} \rightarrow$ Treat
(8) Image $\rightarrow$ Correction $\rightarrow$ Image $\rightarrow$ Treat $\rightarrow$ Image during $\mathrm{Tx} \rightarrow$ Treat $\rightarrow$ Image after Tx

- Thank you for your time - 


\section{Appendix 2}

This patterns-of-care survey is composed of three clinical scenarios related to the experience in the clinical setting. Please select your current practice for each case.

Hospital: $\quad$ Name:

\section{Case 1.}

A 69-year-old male patient was initially diagnosed with prostate cancer with two pelvic bone metastases (Eastern Cooperative Oncology Group [ECOG] 1, Gleason score [G/S] = 4+4, cT4N0M1, initial prostate-specific antigen [PSA] $>1,000 \mathrm{ng} / \mathrm{mL}$, and metastases at the right acetabulum and pubic bone). He received androgen deprivation therapy (ADT) for 1 year. The PSA level decreased to $<0.03 \mathrm{ng} / \mathrm{mL}$ at 6 months after ADT and was maintained. Subsequently, he was referred for radiotherapy (RT). He had no symptoms of bone metastases.

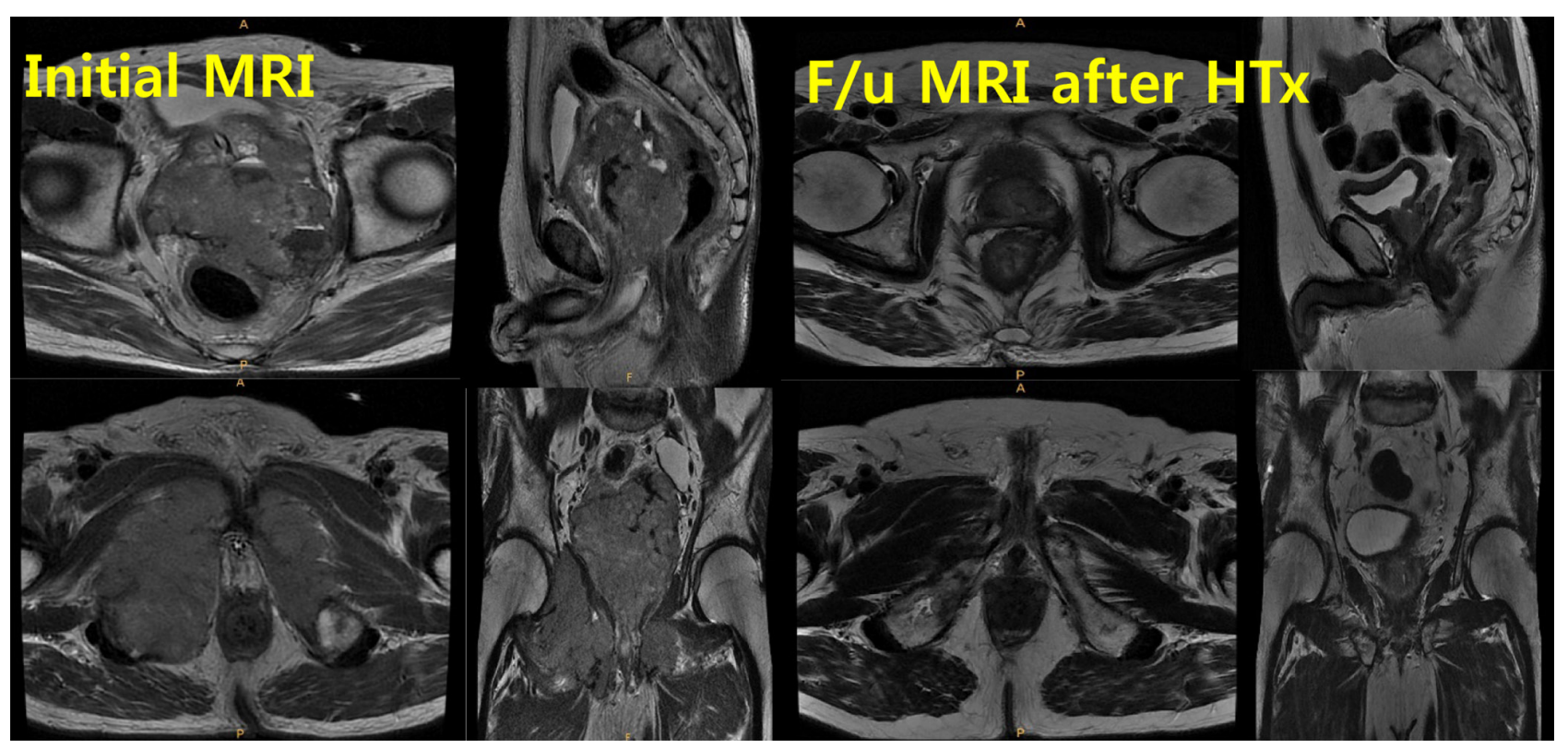

1-1.Does this case correspond with oligometastatic prostate cancer (OMPC)? ( ) Do you agree with the delivery of a high dose in this case? ( )
(1) Yes
(2) No

1-2. Do you have experience with RT to treat prostate cancer patients with limited metastases within the pelvis similar to this? ( )
(1) Yes
(2) No

1-3. When do you initiate RT? ( )

(1) As soon as possible: Move to question 1-4.

(2) No RT: Move to Case 2.

$1-4$. What target volume do you treat for this case? ( )
(1) Prostate only
(2) Whole pelvis including regional lymph node (LN) chains and pelvic bone metastases
(3) Prostate and pelvic bone metastases
(4) Pelvic bone metastases
(5) Others (Please specify) 
1-5. What technique and fractionation scheme do you apply? ( )

Technique type:
(1) $2 \mathrm{D}$
(2) 3DCRT
(3) IMRT
(4) IMRT-SIB
(5) SBRT

A. Prostate: Technique ( ), Fractionation scheme: ( Gy/ fx's)

B. Bone metastases: Technique ( ), Fractionation scheme: ( Gy/ fx's)

C. Whole pelvis: Technique ( ), Fractionation scheme: ( Gy/ fx's)

$\rightarrow$ Followed by prostate boost: Technique ( ), Fractionation scheme: ( Gy/ fx's)

D. Comment:

1-6. What are the reasons why you are not using SBRT for this case? ( ) Can select up to two answers.
(1) Application of whole pelvic RT including elective LN chains
(2) Preference for other fractionation scheme
(3) The lack of special equipment
(4) The lack of experience with the use of SBRT
(5) Insurance problems: limited fractions $(\leq 4)$
(6) Application of other fractionation scheme with generous margin including involved bone metastases
(7) Others (Please specify)

\section{Case 2.}

A 64-year-old male patient was initially diagnosed with prostate cancer with three bone metastases (ECOG 0, G/S $=4+4$, cT2N0M1, initial PSA $162.88 \mathrm{ng} / \mathrm{mL}$, metastases in the left acetabulum, left sacral alar, and $11^{\text {th }}$ thoracic [T11] spine). He received 1 cycle of ADT, and the level of PSA decreased to $41.40 \mathrm{ng} / \mathrm{mL}$ at 1 month after ADT. Subsequently, he was referred for RT. He had no symptoms of bone metastases.

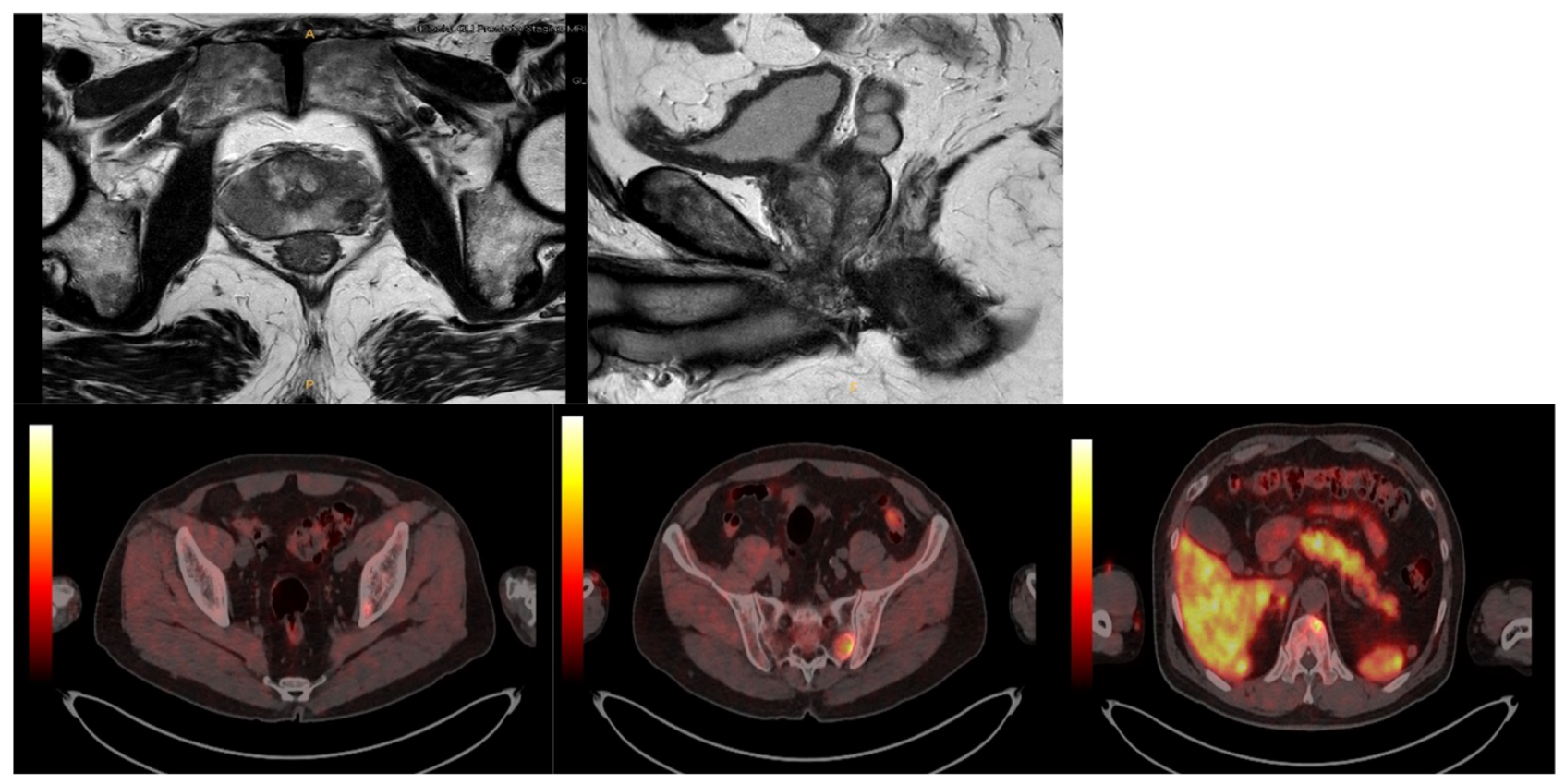

2-1. Does this case correspond to OMPC? ( ) Do you agree with the delivery of a high dose in this case? ( )
(1) Yes
(2) No

2-2. Do you have experience with RT to treat prostate cancer patients with limited bone metastases like this? ( )
(1) Yes
(2) $\mathrm{No}$ 
2-3. When do you initiate RT? ( )

(1) As soon as possible: Move to question 2-4.

(2) Beginning after ( ) months to receive additional ADT as a neoadjuvant aim: Move to question 2-4.

(3) No RT: Move to Case 3.

2-4. What target volume do you treat for this case? ( )

(1) Prostate only

(2) Whole pelvis including regional LN chains and 2 pelvic bone metastases

(3) Prostate and 2 pelvic bone metastases (4) Prostate and 3 bone metastases

(5) Whole pelvis, including regional LN chains and 2 pelvic bone metastases, and T11 spine metastases

(6) 3 bone metastases 7 Others (Please specify)

2-5. What technique and fractionation scheme do you apply? ( )

Technique type:
(1) $2 \mathrm{D}$
(2) 3DCRT
(3) IMRT
(4) IMRT-SIB (5) SBRT

A. Prostate: Technique ( ), Fractionation scheme: ( Gy/ fx's)

B. Bone metastases

- Left acetabulum: Technique ( ), Fractionation scheme: ( Gy/ fx's)

- Left sacral alar: Technique ( ), Fractionation scheme: ( Gy/ fx's)

- T11 spine: Technique ( ), Fractionation scheme: ( Gy/ fx's)

C. Whole pelvis: Technique ( ), Fractionation scheme: ( Gy/ fx's)

$\rightarrow$ Followed by prostate boost: Technique ( ), Fractionation scheme: ( Gy/ fx's)

D. Comment:

2-6. What are the reasons why you are not using SBRT for this case? ( ) Can select up to 2 answers.

(1) Application of whole pelvic RT including elective LN chains (2) Preference for other fractionation scheme

$\begin{array}{ll}\text { (3) The lack of special equipment } & \text { (4) The lack of experience with the use of SBRT }\end{array}$

(5) Insurance problems: limited fractions $(\leq 4)$

(6) Insurance problems: excess of those covered by medical insurance because the RT site is classified as cervical spine/T spine/ lumbar spine/ sacrum.

(7) Application of other fractionation scheme with generous margin including the involved bone metastases

(8) Others (Please specify)

2-7.In what order do you apply RT if you treat both primary lesion and metastatic lesions? ( )

(1) Simultaneous treatment including 3 bone metastases in a day

(2) Simultaneous treatment: Treat 1 site per day in case of bone metastases.

(3) Sequential treatment: Treat all bone metastases in a day after the completion of RT for the primary lesion.

(4) Sequential treatment: Sequentially treat 1 site per day in case of bone metastases after the completion of RT for primary lesion.

(5) Time interval of $>1$ month between RT of primary lesion and RT of bone metastases

(6) Others (Please specify)

\section{Case 3.}

A 65 -year-old male patient was initially diagnosed with prostate cancer with pelvic LN metastases (ECOG 1, G/S $=5+4$, cT3N1M0, initial PSA of $161 \mathrm{ng} / \mathrm{mL}$ ). He was treated with ADT for 4 years. The level of PSA decreased to $0.19 \mathrm{ng} /$ $\mathrm{mL}$ but rebounded to $1.20 \mathrm{ng} / \mathrm{mL}$, and laparoscopic radical prostatectomy was done. Additional ADT was undergone for 3 years. The level of PSA decreased to $0.16 \mathrm{ng} / \mathrm{mL}$ but rebounded to $1.08 \mathrm{ng} / \mathrm{mL}$, and a salvage RT to prostate bed with $70 \mathrm{~Gy} / 35$ fx's was done. Duo to the continuous increase in the level of PSA, he received 4 cycles of docetaxel plus prednisone, but this treatment was discontinued due to the occurrence of neutropenia. The level of PSA at follow-up was $7.89 \mathrm{ng} / \mathrm{mL}$, and single spine metastases at the T12 was detected on bone scan. Subsequently, he was referred for RT. He had no symptoms of bone metastases. 

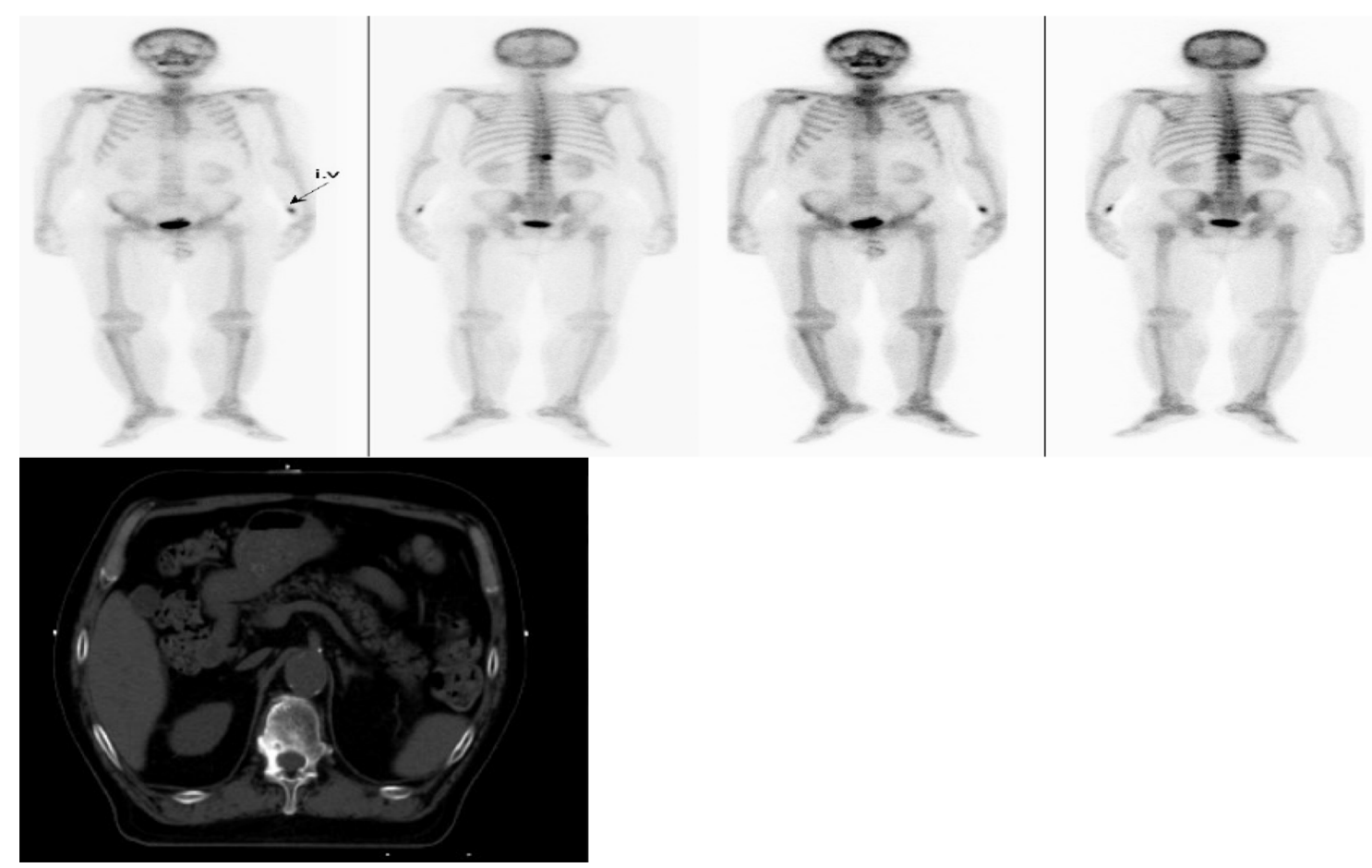

3-1.Does this case correspond to OMPC? ( ) Do you agree with the delivery of a high dose in this case? ( )
(1) Yes
(2) No

3-2. Do you have experience with RT to treat prostate cancer patients with solitary metastases similar to this? ( )

(1) Yes (2) No

3-3. When do you initiate RT? ( )

(1) As soon as possible: Move to question 3-4.

(2) No RT: End the survey and thank you for your time.

3-4. What target volume do you treat for this case? ( )
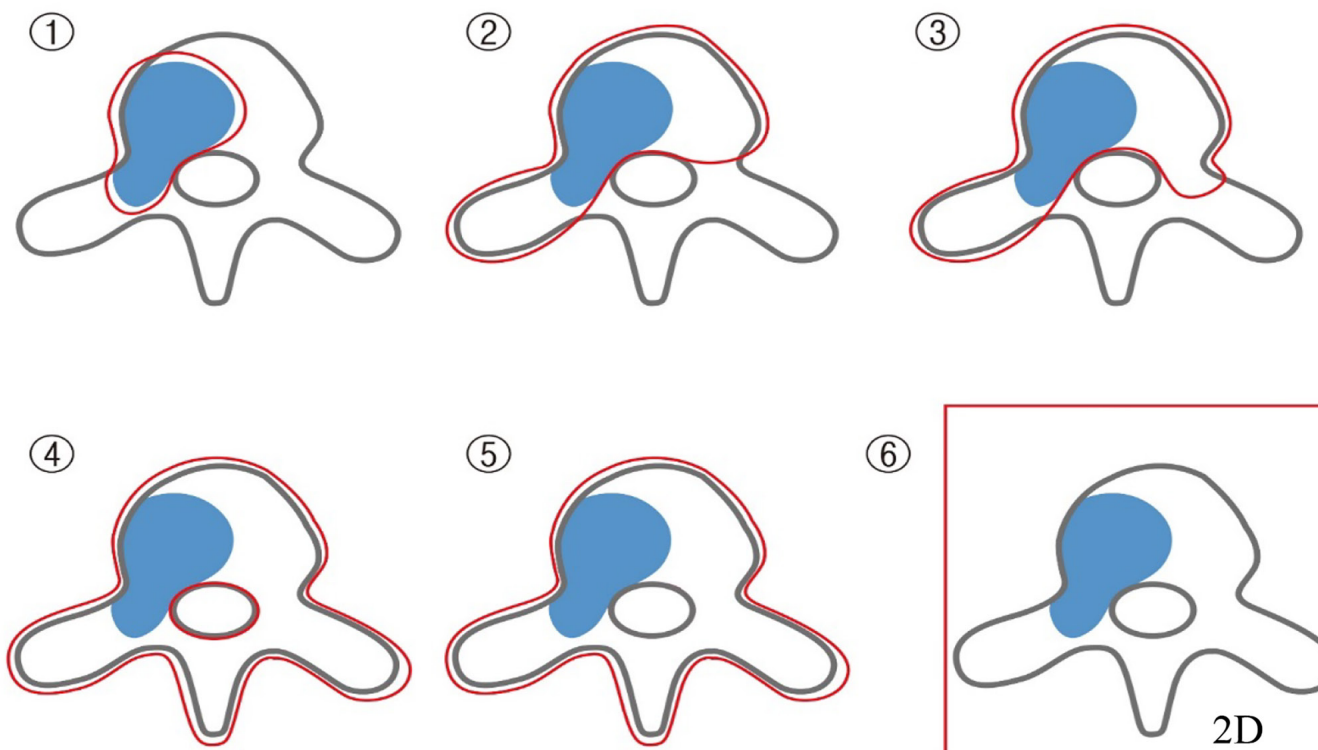

(6)

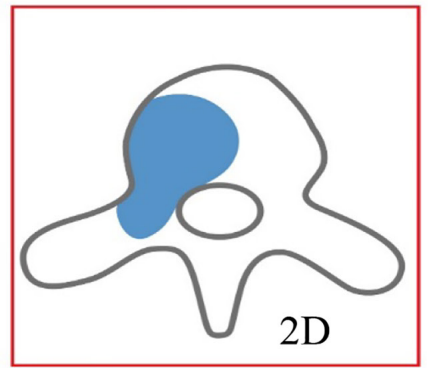


3-5. What technique and fractionation scheme do you apply? ( )

Technique type ( ):
(1) $2 \mathrm{D}$
(2) 3DCRT
(3) IMRT
(4) IMRT-SIB
(5) SBRT

Fractionation scheme: ( Gy/ fx's)

3-6. What are the reasons why you do not use SBRT for this case? ( ) Can select up to 2 answers.

(1) Preference for other fractionation scheme

(2) The lack of special equipment

(3) The lack of experience with the use of SBRT

(4) Insurance problems: limited fractions $(\leq 4)$

(5) Application of other fractionation scheme with generous margin including involved bone metastases

(6) Others (Please specify)

- Thank you for your time -

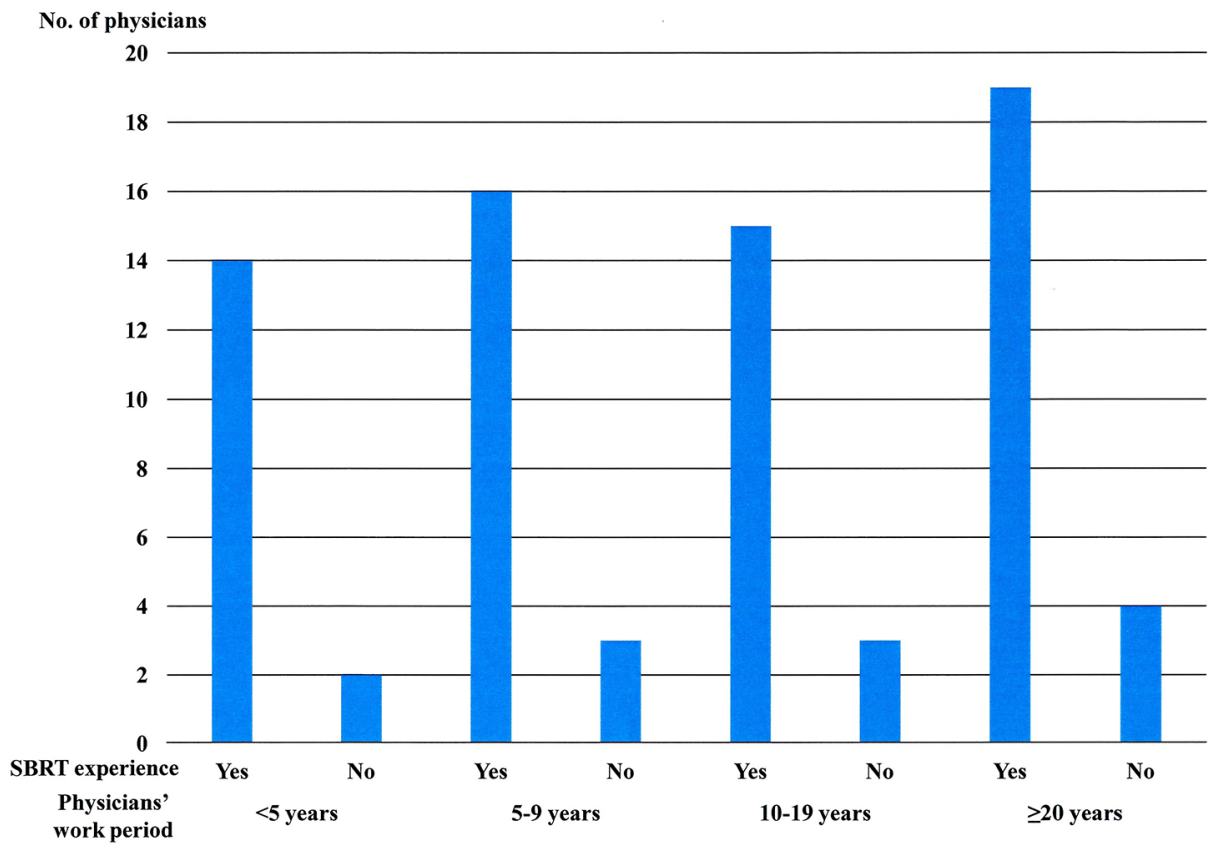

Figure S1 Stereotactic body radiotherapy (SBRT) experience according to physicians' work period. 